\title{
US Primary Care Physicians' Viewpoints on HPV Vaccination for Adults 27 to 45 Years
}

\author{
Laura P. Hurley, MD, MPH, Sean T. O'Leary, MD, MPH, Lauri E. Markowitz, MD, \\ Lori A. Crane, PhD, MPH, Jessica R. Cataldi, MD, MSCS, \\ Michaela Brtnikova, PhD, MPH, Brenda L. Beaty, MSPH, Carol Gorman, BA, \\ Elissa Meites, MD, MPH, Megan C. Lindley, MPH, and Allison Kempe, MD, MPH
}

Introduction: In June 2019, the Advisory Committee on Immunization Practices (ACIP) recommended shared clinical decision making (SCDM) regarding human papillomavirus (HPV) vaccination for adults 27 to 45 years. Our objectives were to assess among primary care physicians 1) recent practice regarding HPV vaccination for adults 27 to 45 years, 2) knowledge of HPV and the new SCDM recommendation, and 3) attitudes toward and anticipated effect of the new SCDM recommendation.

Methods: From October to December 2019, we administered an Internet and mail survey to national networks of 494 general internist (GIM) and 474 family physician (FP) members of the American College of Physicians and American Academy of Family Physicians, respectively.

Results: Response rate was 64\% (617/968; GIM, 57\%; FP, 71\%). Fifty-eight percent were aware of the new ACIP recommendation; $42 \%$ had recommended HPV vaccination to adults 27 to 45 years, but most had administered HPV vaccine to very few of these patients (73\% to $0 \%$ and $22 \%$ to 1 to 3$)$. Fiftyfive percent and $63 \%$ were unaware that HPV vaccination does not prevent progression of existing HPVrelated cancers or infections, respectively and $57 \%$ were not sure what to emphasize when having a SCDM conversation about HPV vaccination. A majority reported they will be more likely recommend HPV vaccination to adults in the 27-to-45-year age range as a result of the new recommendation.

Conclusions: Physicians are interested in recommending HPV vaccination for adults age 27 to 45 years despite ACIP not routinely recommending it in this age range. The majority need more education about the optimal use of HPV vaccine in this age group. (J Am Board Fam Med 2021;34:162-170.)

Keywords: Cancer, Family Physicians, Immunization, Papillomavirus Infections, Papillomavirus Vaccines, Primary Health Care, Surveys and Questionnaires

\section{Introduction}

Human papillomavirus (HPV) is the most common sexually transmitted infection in the United States. ${ }^{1}$ Most HPV infections are asymptomatic and resolve

This article was externally peer reviewed.

Submitted 3 August 2020; revised 18 September 2020; accepted 21 September 2020.

From Adult and Child Consortium for Health Outcomes Research and Delivery Science, University of Colorado Anschutz Medical Campus and Children's Hospital Colorado, Aurora, CO (LPH, STO, LAC, JRC, MB, BLB, CG, AK); Division of General Internal Medicine, Denver Health, Denver, CO (LPH); Department of Pediatrics, Children's Hospital-Colorado, Aurora, CO (STO, JRC, $\mathrm{MB}, \mathrm{AK})$; National Center for Immunization and Respiratory Diseases, Centers for Disease Control and Prevention, Atlanta, GA (LEM, EM, MCL); Department of Community and Behavioral Health, University of Colorado Anschutz Medical Campus, Aurora, CO (LAC).

Funding: This publication was supported by Cooperative Agreement Number 1 U01 IP000849-03, funded by the spontaneously; nonetheless, some will become chronic and can cause genital warts and various types of cancer, most frequently oropharyngeal, cervical, or anal cancer, usually decades after initial infection. ${ }^{2}$ Each year approximately 34,800 new cases of HPV-attributable cancer occur in the United States. $^{3}$

Since 2006, 3 different HPV vaccines have been approved for use in the United States. A 9-valent $\mathrm{HPV}$ vaccine $(9 \mathrm{vHPV})$, which is currently the only HPV vaccine distributed in the United States,

Centers for Disease Control and Prevention. The findings and conclusions in this report are those of the authors and do not necessarily represent the official position of the Centers for Disease Control and Prevention.

Conflict of interest: None.

Corresponding author: Laura P. Hurley, MD, MPH, 301 W. 6th Avenue, MC3251, Denver, CO 80204 (E-mail: Laura.Hurley@dhha.org). 
protects against HPV types that cause $90 \%$ of genital warts and $92 \%$ of HPV-related cancers. ${ }^{3}$ Until recently, 9vHPV was only licensed for use in adolescents and young adults less than 27 years. ${ }^{4-7}$

However, some men and women remain at risk for new HPV infections throughout their sexually active lives and HPV vaccines have been shown to be immunogenic in men age 27 to 45 years and to prevent persistent infection and HPV-related precancerous lesions in women age 27 to 45 years. ${ }^{8-13}$ Countries have had different approaches in terms of licensing HPV vaccines and recommending $\mathrm{HPV}$ vaccination in this age range. For example, although China, Australia, Canada, and many European countries have licensed HPV vaccines through age 45 years, their national immunization programs have targeted younger age groups; outside of targeted groups, vaccination costs are either not covered or incompletely covered by national health insurance programs. ${ }^{14-16}$

In October 2018, the US Food and Drug Administration expanded the licensed age range for $9 \mathrm{vHPV}$ in the United States from 9 to 26 years to 9 to 45 years. $^{17}$ In June 2019, the Advisory Committee on Immunization Practices (ACIP) did not extend the recommendation for catch-up HPV vaccination to include adults age 27 to 45 years, but instead, noting that some adults who are not adequately vaccinated might be at risk for new HPV infections and might benefit from vaccination in this age range, recommended shared clinical decision making (SCDM) regarding potential HPV vaccination. ${ }^{18}$ Most health insurance should cover the cost of vaccination that occurs as a result of SCDM recommended by ACIP, under provisions of the Affordable Care Act. ${ }^{19}$ Formal publication of these recommendations in August 2019 included 8 talking points for physicians to consider when having a SCDM conversation with adults age 27 to 45 years about receiving HPV vaccination, including epidemiology of HPV infections and disease, vaccine efficacy and effectiveness in various age groups, characteristics of HPV vaccines including their expected prophylactic rather than therapeutic effects, and that no antibody test is available to determine whether someone is still susceptible to HPV infections that the vaccination can prevent. ${ }^{18}$

Previous surveys of predominately insured American women in this age range have found that most would choose to receive HPV vaccination if it was approved for use in this age range. ${ }^{20,21}$
Because a physician recommendation has been identified as an important predictor of receipt of vaccinations among adults in general ${ }^{22-25}$ and HPV vaccination in particular, ${ }^{26}$ the objectives of this study were to assess among US primary care physicians 1) recent practice regarding HPV vaccination in adults age 27 to 45 years, 2) knowledge of HPV and the new SCDM recommendation, and 3) attitudes toward and anticipated effect of the new SCDM recommendation.

\section{Methods \\ Study Setting}

From October 2019 to January 2020, a survey was administered to a national network of physicians who spent at least $50 \%$ of their time practicing primary care. The human subjects review board at the University of Colorado Anschutz Medical Campus approved this study as exempt research.

\section{Study Population}

The Vaccine Policy Collaborative Initiative, a survey entity to assess physician practice and attitudes about vaccines and vaccine policy, working with the Centers for Disease Control and Prevention, developed and conducted the survey among physician networks designed to be representative of the American College of Physicians and the American Academy of Family Physician memberships. ${ }^{27-28}$ These physician networks are similar to physicians randomly sampled from the American Medical Association Masterfile with respect to demographics and attitudes regarding vaccines. ${ }^{27}$

\section{Study Design}

The survey asked about whether HPV vaccines were stocked in the practice, and about physician behavior regarding recommending the vaccine for various groups of adults age 27 to 45 years: 1) healthy adults, 2) those with an immunocompromising condition, 3) those with a new sex partner, 4) those who have multiple sex partners, 5) those in a mutually monogamous sexual relationship, and 6) men who have sex with men. The survey assessed frequency in the past 3 months patients in this age range had requested HPV vaccination ("never," "rarely," "sometimes," "almost always/always") and how often physicians had given it (" 0, , " 1 to 3, , "4 to 9," and "10 or more"). Physicians were asked about the types of patients age 27 to 45 years who 
had requested it (worried adults who have low risk for HPV-related disease, adults at high risk for HPV-related disease, adults who are planning to have a new sex partner, and adults who already have an HPV-related infection or disease). Physicians were presented with a series of true and false questions aimed to assess knowledge of HPV disease and vaccination and including a "donot-know" response option. Four-point Likert scales were used to assess physician attitudes regarding HPV vaccination for adults age 27 to 45 years ("strongly agree" to "strongly disagree"). Physicians were provided the following information: The ACIP did not recommend routine catch-up vaccination for adults age 27 to 45 years. However, because some adults in this age range are at risk for new HPV infection and might benefit from vaccination, ACIP recommended SCDM for these individuals, and the survey asked about prior awareness of this recommendation. Physicians were then asked how the new recommendation affects how they will recommend HPV vaccination to adults in this age range in the future, with 3 response options ("I will be less likely to recommend," "My recommendation will not change," and "I will be more likely to recommend"). A national advisory panel of general internists (GIM) $(\mathrm{n}=3)$ and family physicians $(\mathrm{FP})(\mathrm{n}=4)$ pretested the survey, which was then modified based on feedback. The survey was then pilot tested among 60 GIM and 8 FP nationally and further modified based on their feedback.

\section{Survey Administration}

Per individual physician preference, the survey was sent by e-mail or through US mail. The e-mail group was sent an initial e-mail and up to 8 e-mail reminders, and the US mail group was sent an initial mailing and up to 2 additional reminders. Nonrespondents in the e-mail group were also sent up to 2 surveys by US mail. Due to an initial low response rate, GIM nonresponders received an additional reminder in a hand-written envelope. ${ }^{28}$ The mail protocol was patterned on Dillman's Tailored Design Method. ${ }^{29}$ The survey was administered 4 months after the ACIP vote in June 2019 and 2 months after formal publication of the recommendation for SCDM regarding HPV vaccination of adults age 27 to 45 years. ${ }^{18}$

\section{Statistical Analysis}

Results were similar by specialty so are presented together, with significant differences highlighted. Respondents and nonrespondents were compared on all available characteristics using t-test, chisquared tests, and Wilcoxon tests, as appropriate. Chi-squared tests were used to compare responses over all response categories between specialties and to assess the potential associations between knowledge of the new ACIP recommendation and having recommended HPV vaccination in the past. Chisquared tests and Fisher exact tests were used to compare physicians who had and who had not recommended HPV vaccination to adults age 27 to 45 years in the past and likelihood of doing so based on the new ACIP recommendation. All analyses were performed using SAS, version 9.4 (SAS Institute, Cary, NC).

\section{Results}

The overall response rate was 64\% (617/968); 71\% (336/474) for FP and 57\% (281/494) for GIM. Characteristics of respondents and nonrespondents are shown in Table 1. Respondents and nonrespondents did not differ by census location (urban, suburban, or rural), region, or whether decisions regarding purchasing and handling of vaccines by the practice were made independently or at a larger system level. Male and older physicians and physicians from private and smaller practices were less likely to respond.

\section{Recent Practice Regarding HPV Vaccination for Adults Age 27 to 45 Years}

Seventy-four percent of respondents reported that $9 \mathrm{vHPV}$ was stocked in their practice (FP, $83 \%$ and GIM, 64\%; $\mathrm{P}<.0001$ for difference between specialties). Forty-two percent of physicians had recommended HPV vaccination to adults age 27 to 45 years in the past; $35 \%$ to adults with multiple sex partners, $33 \%$ to men who have sex with men, $29 \%$ to adults with a new sex partner, $27 \%$ to adults with an immunocompromising condition, and $15 \%$ to adults in a mutually monogamous sexual relationship. Seventy-three percent had not administered $\mathrm{HPV}$ vaccine to any adult in this age range in the past 3 months; $22 \%$ had administered to 1 to 3 patients, $4 \%$ to 4 to 9 patients, and only $1 \%$ had administered to $>10$ patients. 
Table 1. Demographic and Practice Characteristics of Survey Respondents and Non-Respondents, United States, 2019-2020 ( $\mathrm{n}=968)$

\begin{tabular}{|c|c|c|c|}
\hline Characteristic & Respondents $(n=617)$ & Non-Respondents $(\mathrm{n}=351)$ & P-Value \\
\hline Mean (SD) physician age, years & $54.8(9.4)$ & $56.7(9.1)$ & $.002 * \dagger$ \\
\hline Female, \% & 50 & 38 & $<.001^{*}$ \\
\hline \multicolumn{4}{|l|}{ Specialty, \% } \\
\hline Family physician & 54 & 39 & \multirow[t]{2}{*}{$<.001^{*}$} \\
\hline General internist & 46 & 61 & \\
\hline \multicolumn{4}{|l|}{ Region of the United States, \% } \\
\hline Midwest & 24 & 26 & \multirow[t]{4}{*}{.08} \\
\hline Northeast & 19 & 21 & \\
\hline South & 33 & 36 & \\
\hline West & 24 & 17 & \\
\hline \multicolumn{4}{|l|}{ Location of practice, $\%$} \\
\hline Urban & 27 & 23 & \multirow[t]{3}{*}{.19} \\
\hline Suburban & 67 & 68 & \\
\hline Rural & 6 & 9 & \\
\hline \multicolumn{4}{|l|}{ Practice setting, \%‡ } \\
\hline Private practice & 66 & 78 & \multirow[t]{3}{*}{$<.001^{*}$} \\
\hline Hospital or clinic & 25 & 17 & \\
\hline Health maintenance organization & 9 & 5 & \\
\hline Median (IQR) number of providers in practice & $6(3$ to 12$)$ & $5(2$ to 10$)$ & $.002 * \$$ \\
\hline \multicolumn{4}{|c|}{ Decisions are made about purchasing and handling vaccines, \% } \\
\hline Independently & 53 & 57 & \multirow[t]{2}{*}{.28} \\
\hline At a larger system level & 47 & 43 & \\
\hline
\end{tabular}

$\mathrm{IQR}$, interquartile range; SD, standard deviation.

*Bold values indicate statistical significance $\mathrm{P}<.05$ between respondents and non-respondents.

${ }^{\dagger}$ t-test used.

${ }^{\ddagger}$ Numbers may not add to $100 \%$ due to rounding.

${ }^{\S}$ Wilcoxon test used.

\section{Physician Perception of Patients Age 27 to 45 Years Requesting HPV Vaccine}

Regarding how often their patients age 27 to 45 years had requested HPV vaccination in the past 3 months without the physician bringing it up, $5 \%$ of physicians reported "sometimes," $25 \%$ reported "rarely," and 70\% reported "never." GIM were more likely than FP to report receiving requests (8\% sometimes/21\% rarely for GIM vs $3 \%$ sometimes $/ 27 \%$ rarely for $\mathrm{FP} ; \mathrm{P}=.02$ for the difference between specialties over all response categories). Respondents with patients 27 to 45 years who had requested an HPV vaccination $(n=186)$, reported that the following types of patients had requested it: adults at high risk for HPV-related disease (57\%), worried adults at low risk for HPV-related disease (53\%), adults who already had an HPV-related infection or disease $(48 \%)$, and adults who were planning to have a new sex partner (41\%).

\section{Physician Knowledge of HPV Disease and Vaccination}

Almost all physicians stated correctly that adults who have a new partner may be at risk of acquiring a new HPV infection (Table 2). A majority knew the vaccination series consists of 3 doses when started as an adult, the series can be completed with $9 \mathrm{vHPV}$ even if initiated with a different HPV vaccine, there is no HPV antibody testing needed before vaccination and ACIP does not recommend against vaccinating adults with multiple sex partners or who have a diagnosed HPV infection. A majority did not know that HPV vaccination does not prevent progression of existing HPV-related cancers or infections and that HPV vaccine effectiveness is lower in adults age 27 to 45 years compared with adults age 18 to 26 years. More FP than GIM answered correctly that when the HPV vaccination series is started with a different HPV vaccine, the series can be completed with 9vHPV (71\% FP vs 
Table 2. Physician Knowledge of HPV Disease and HPV Vaccination, United States, 2019 to $2020(n=617)$

\begin{tabular}{|c|c|c|c|c|}
\hline Knowledge Concept: True or False? & $\begin{array}{l}\text { Correct } \\
\text { Answer }\end{array}$ & $\begin{array}{c}\text { Physicians } \\
\text { Answering } \\
\text { Correctly (\%) }\end{array}$ & $\begin{array}{c}\text { Physicians } \\
\text { Answering } \\
\text { Incorrectly (\%) }\end{array}$ & $\begin{array}{c}\text { Physicians } \\
\text { Answering, "Don't } \\
\text { Know" (\%) }\end{array}$ \\
\hline $\begin{array}{l}\text { Adults who have a new sex partner are at risk of acquiring a new } \\
\text { HPV infection }\end{array}$ & True & 96 & 1 & 3 \\
\hline $\begin{array}{l}\text { ACIP recommends against } 9 \mathrm{vHPV} \text { in unvaccinated adults age } 27 \\
\text { through } 45 \text { years who have had multiple sex partners }\end{array}$ & False & 70 & 11 & 19 \\
\hline $\begin{array}{l}\text { People who started the HPV vaccination series with an older } \\
\text { vaccine can complete the series with } 9 \mathrm{vHPV}^{*}\end{array}$ & True & 65 & 6 & 30 \\
\hline $\begin{array}{l}\text { HPV antibody testing is recommended for adults age } 27 \text { through } \\
45 \text { years as a part of shared clinical decision making for HPV } \\
\text { vaccination }\end{array}$ & False & 64 & 13 & 23 \\
\hline $\begin{array}{l}\text { Full HPV vaccination of an adult who started the series as an adult } \\
\text { will require three doses of } 9 \mathrm{vHPV} *\end{array}$ & True & 56 & 21 & 24 \\
\hline $\begin{array}{l}\text { ACIP recommends against } 9 \mathrm{vHPV} \text { in unvaccinated adults age } 27 \\
\text { through } 45 \text { years who have had a diagnosed HPV infection }\end{array}$ & False & 55 & 10 & 35 \\
\hline HPV vaccine prevents progression of existing HPV related cancers & False & 45 & 17 & 38 \\
\hline HPV vaccine prevents progression of existing HPV infection & False & 38 & 22 & 41 \\
\hline $\begin{array}{l}\text { HPV vaccine effectiveness is lower for adults age } 27 \text { through } \\
45 \text { years compared to adults age } 18 \text { through } 26 \text { years }\end{array}$ & True & 30 & 30 & 41 \\
\hline $\begin{array}{l}\text { Studies show that HPV vaccine is very effective in adults age } 27 \\
\text { through } 45 \text { years with HIV infection }\end{array}$ & False & 8 & 37 & 56 \\
\hline
\end{tabular}

Some percentages do not add up to $100 \%$ because of rounding.

${ }^{*} P \leq .05$ for statistical differences between specialties. Seventy-one percent of FP and $57 \%$ of GIM answered correctly that when HPV vaccination series was started with an older vaccine, it can be completed with 9vHPV. Fifty-nine percent of FP and $51 \%$ of GIM were aware that when HPV vaccination started as an adult requires 3 doses.

HPV, Human Papillomavirus; ACIP, Advisory Committee on Immunization Practices; HIV, human immunodeficiency virus.

57\% GIM; P $\leq .001$ ) and that when HPV vaccination is started as an adult 3 doses are recommended ( $59 \% \mathrm{FP}$ vs $51 \%$ of GIM; $\mathrm{P}=.03)$.

\section{Physician Knowledge and Attitudes Regarding SCDM Recommendation for HPV Vaccination}

Before reading the information provided in the survey, $58 \%$ of respondents were aware of the recommendation for SCDM regarding HPV vaccination of adults age 27 to 45 years. Knowledge of the ACIP recommendation was associated with having recommended HPV vaccination to an adult in this age group in the past $(59 \%$ of those aware had recommended in the past vs $19 \%$ who were unaware, $\mathrm{P} \leq .001)$. Over half of physicians (16\% strongly agreed, $41 \%$ somewhat agreed) were unsure of what points to emphasize when having a SCDM conversation about HPV vaccination with an adult age 27 to 45 years. GIM were more likely to be unsure of what points to emphasize (GIM 19\% strongly agreed/ $44 \%$ somewhat agreed vs FP $13 \%$ strongly $/ 38 \%$ somewhat agreed, $\mathrm{P}=.01)$.

\section{Anticipated Effect of ACIP Recommendation on Physician Recommendation for HPV Vaccination in Adults Age 27 to 45 Years}

Figure 1 shows how the ACIP recommendation would affect physicians' practices regarding HPV vaccination for particular groups overall and stratified by whether physicians had already recommended HPV vaccination to those groups. Almost all physicians who had recommended HPV vaccination to adults age 27 to 45 years would either not change behavior (ie, would continue to recommend) or would be more likely to recommend HPV vaccination to adults in this age range. Compared with physicians who had recommended HPV vaccination previously, more physicians who had not done so previously were now more likely to recommend $\mathrm{HPV}$ vaccination for all groups of adults in this age range $(\mathrm{P}<.001)$, except to healthy adults without known risk factors, and to adults in a mutually monogamous sexual relationship. Only $5 \%$ of physicians had not recommended HPV vaccination to any group and were not planning to change behavior (ie, would continue to not recommend). 
Figure 1. Anticipated changes in physician practice following ACIP recommendation for SCDM regarding HPV vaccination of adults 27 to 45 years, United States, 2019 to 2020 ( $n=534)$. Abbreviations: ACIP, Advisory Committee on Immunization Practices; SCDM, shared clinical decision making; HPV, Human Papillomavirus.

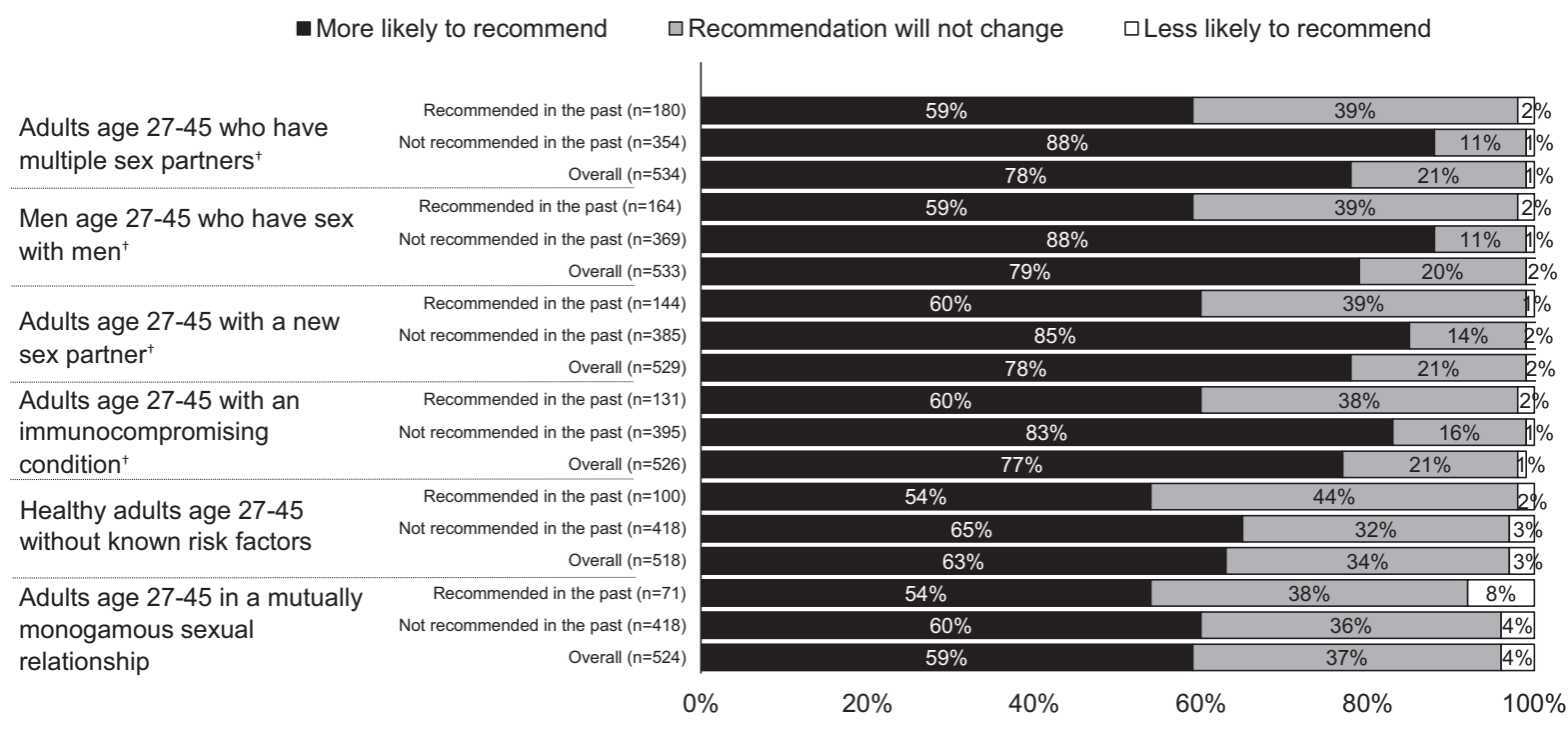

"Some percentages do not add up to $100 \%$ due to rounding

†More physicians who had not recommended than who had recommended HPV vaccination to adults in this group would be more likely to recommend it now, $p=0.001-45$

\section{Discussion}

This is the first study to evaluate the physician perspective on the new ACIP recommendation regarding HPV vaccination for adults age 27 to 45 years. Although the data show that nearly half of physicians had recommended HPV vaccination to an adult in this age group, most had not yet administered it in the several months after the new recommendation was made and published. Fifty-eight percent of physicians knew of the SCDM recommendation and knowledge of this recommendation was associated with having recommended HPV vaccination to an adult in this age range. The vast majority of surveyed physicians, regardless of their previous practices regarding adults in this age range, reported the new recommendation would increase their likelihood of recommending HPV vaccination to adults age 27 to 45 years.

The majority of physicians were knowledgeable about other aspects of HPV vaccination recommendations (Table 2); however, some were not aware the vaccination series can be completed with 9vHPV even if initiated with another HPV vaccine, the recommended series for adults initiating vaccination is 3 doses, and that antibody testing is not recommended. FPs were slightly more knowledgeable than
GIM about other vaccination recommendations, likely as a result of caring for adolescents for whom there has been a longstanding routine recommendation for $\mathrm{HPV}$ vaccination.

Gaps in knowledge of HPV disease and vaccination identified here indicate physicians may be overestimating the benefits of HPV vaccination for some populations. Although HPV vaccination is highly effective when given before first exposure to HPV, HPV vaccination does not prevent the progression of existing HPV infections or disease..$^{30,31}$ The finding that only $38 \%$ of physicians knew HPV vaccine does not prevent the progression of HPV infection are in contrast to a 2010 study of Australian primary care physicians which showed almost all (95\%) were aware of this. ${ }^{32}$ In addition, many physicians were not aware HPV vaccine effectiveness is lower in adults age 27 to 45 years than in younger individuals. Because many adults have already been exposed to HPV, vaccine effectiveness is lower with increasing age. Thus, the public health benefit of HPV vaccination over age 26 years is expected to be minimal. ${ }^{18}$

Knowledge gaps among physicians may make it challenging for them to have a SCDM conversation with adults about HPV vaccination and likely 
contribute to physicians being unsure of what to emphasize during such conversations. There are several other factors to consider as well. First, to inform a SCDM conversation, physicians need to ascertain sexual history. Although sexual history taking by primary care physicians has not been extensively studied, at least one study, conducted before any ACIP HPV vaccination recommendations, indicated that almost half of physicians did not take a sexual history as part of an annual examination; very few physicians asked about sexual history during every visit. ${ }^{33}$ Second, it is inherently difficult to know which adults might benefit from HPV vaccination because this depends on both past and future exposures to vaccine-type HPV through sexual activity. This may be one reason why physicians, particularly those who had previously recommended HPV vaccination for adults in this age range, did not make specific distinctions in their recommendations to various risk groups. Third, SCDM conversations may be viewed as potentially time-consuming, a challenge when there are time constraints during a primary care visit as the complexity and number of tasks for physicians to address per visit has increased. ${ }^{34,35}$

Our findings suggest most physicians plan to recommend HPV vaccination to at least some adults in this age group. The ACIP recommendation states, "HPV vaccination does not need to be discussed with most adults aged $>26$ years." ${ }^{18}$ This is an important consideration as there is a global HPV vaccine shortage. ${ }^{36}$ The focus should remain on vaccinating adolescent and young adult populations who are most likely to benefit; to date, the US $\mathrm{HPV}$ vaccination program, targeting these younger groups, has been successful at reducing vaccinetype HPV infections and related diseases ${ }^{37}$ and has been cost effective and potentially even cost saving. ${ }^{38}$ Extending vaccination to all adults age 27 to 45 years is predicted to have little impact on morbidity and mortality, resulting in considerably less favorable cost effectiveness ratios than vaccinating younger populations. ${ }^{39}$

Although this survey includes nationally representative samples of primary care physicians who care for adults in the United States and the response rate was high, the findings are subject to several limitations. Males; older physicians; and physicians from smaller, private practices are somewhat underrepresented in these findings and thus may limit the generalizability of the results. There may be an element of social desirability bias in terms of physicians reporting they will recommend this vaccine even though the survey included information stating that ACIP does not routinely recommend HPV vaccination for adults. The report of likelihood of vaccinating may not translate into actually vaccinating, and the number of adults anticipated to be vaccinated could not be quantified from the survey. In addition, we did not ask physicians specifically about having a SCDM conversation about HPV vaccination.

Primary care physicians, key stakeholders in the acceptability of HPV vaccination among adults 27 to 45 years, expressed great interest in vaccinating this age-group against HPV, despite the lack of a routine recommendation from ACIP. Addressing knowledge deficits identified in this survey could assist physicians in implementing a recommendation for SCDM with their patients. Development of a decision aid, which has been suggested to improve the quality of SCDM,${ }^{40}$ may be helpful in prompting physicians to assess relevant patient history, to cull the 8 talking points provided in the ACIP recommendation, and clearly convey knowledge concepts and thus support physicians having conversations with adults age 27 to 45 years about HPV vaccination.

The authors thank Selam Wubu, MPH; and Darilyn Moyer, MD from the ACP; and Amy Mullins, MD; and Bellinda Schoof, MHA from the AAFP for collaborating in the establishment of the sentinel networks in general internal medicine and family medicine. We would also like to thank all general internists and family physicians in the networks for participating and responding to this survey.

To see this article online, please go to: http://jabfm.org/content/ 34/1/162.full.

\section{References}

1. Satterwhite CL, Torrone E, Meites E, et al. Sexually transmitted infections among US women and men: prevalence and incidence estimates, 2008. Sex Transm Dis 2013;40:187-93.

2. Centers for Disease Control and Prevention. HPV diseases and cancers. 2019. Available from: https:// www.cdc.gov/hpv/parents/cancer.html. Accessed April 15, 2020.

3. Senkomago V, Henley SJ, Thomas CC, Mix JM, Markowitz LE, Saraiya M. Human papillomavirusAttributable Cancers-United States, 2012-2016. MMWR Morb Mortal Wkly Rep 2019;68:724-8. Available from: https://www.cdc.gov/mmwr/volumes/ 68/wr/mm6833a3.htm. Accessed May 5, 2020. 
4. American College of Obstetricians and Gynecologists. Committee opinion No. 641: human papillomavirus vaccination. Obstetr Gynecol 2015;126:e38-e43.

5. Markowitz LE, Dunne EF, Saraiya $M$, et al. Human papillomavirus vaccination: recommendations of the Advisory Committee on Immunization Practices (ACIP). MMWR Morb Mortal Wkly Rep. 2014;63(Rr-05):1-30.

6. Centers for Disease Control and Prevention. Recommendations on the use of quadrivalent human papillomavirus vaccine in males-Advisory Committee on Immunization Practices (ACIP), 2011. MMWR Morb Mortal Wkly Rep 2011;60: 1705-8.

7. Markowitz LE, Dunne EF, Saraiya M, Lawson HW, Chesson H, Unger ER. Quadrivalent human papillomavirus vaccine: recommendations of the Advisory Committee on Immunization Practices (ACIP). MMWR Recomm Rep 2007;56:1-24.

8. Castellsague X, Munoz N, Pitisuttithum P, et al. End-of-study safety, immunogenicity, and efficacy of quadrivalent HPV (types 6,11, 16, 18) recombinant vaccine in adult women 24-45 years of age. $\mathrm{Br}$ J Cancer 2011;105:28-37.

9. Munoz N, Manalastas R, Jr, Pitisuttithum P, et al. Safety, immunogenicity, and efficacy of quadrivalent human papillomavirus (types 6, 11, 16, 18) recombinant vaccine in women aged $24-45$ years: a randomised, double-blind trial. Lancet (London, England) 2009;373:1949-57.

10. Wei L, Xie X, Liu J, et al. Efficacy of quadrivalent human papillomavirus vaccine against persistent infection and genital disease in Chinese women: a randomized, placebo-controlled trial with 78month follow-up. Vaccine 2019;37:3617-24.

11. Skinner SR, Szarewski A, Romanowski B, et al. Efficacy, safety, and immunogenicity of the human papillomavirus 16/18 AS04-adjuvanted vaccine in women older than 25 years: 4-year interim followup of the phase 3, double-blind, randomised controlled VIVIANE study. Lancet (London, England) 2014;384:2213-27.

12. Wheeler CM, Skinner SR, Del Rosario-Raymundo MR, et al. Efficacy, safety, and immunogenicity of the human papillomavirus 16/18 AS04-adjuvanted vaccine in women older than 25 years: 7 -year follow-up of the phase 3, double-blind, randomised controlled VIVIANE study. The Lancet Infectious diseases 2016;16:1154-68.

13. Giuliano AR, Isaacs-Soriano $\mathrm{K}$, Torres BN, et al. Immunogenicity and safety of Gardasil among midadult aged men (27-45 years) - The MAM Study. Vaccine 2015;33:5640-6.

14. Government of Canada. Update on the recommended Human Papillomavirus (HPV) vaccine immunization schedule. 2016. Available from: https://www.canada. ca/en/public-health/services/publications/healthyliving/update-recommended-human-papillomavirus- vaccine-immunization-schedule.html. Accessed April 17,2020 .

15. Mazza D, Petrovic K, Grech C, Harris N. HPV vaccination in women aged 27 to 45 years: what do general practitioners think? BMC Womens Health 2014;14:91.

16. Lin $\mathrm{Y}$, Lin $\mathrm{Z}, \mathrm{He} \mathrm{F}$, et al. HPV vaccination intent and willingness to pay for 2-,4-, and 9-valent HPV vaccines: a study of adult women aged $27-45$ years in China. Vaccine 2020;38:3021-30.

17. US Department of Health and Human Services FaDA. Gardasil 9 (human papillomavirus 9-valent vaccine, recombinant). Washington, DC: Health and Human Services FaDA; 2018.

18. Meites E, Szilagyi PG, Chesson HW, Unger ER, Romero JR, Markowitz LE. Human papillomavirus vaccination for adults: updated recommendations of the Advisory Committee on Immunization Practices. MMWR Morb Mortal Wkly Rep 2019;68:698-702.

19. Centers for Disease Control and Prevention. ACIP Shared Clinical Decision-Making Recommendations. 2020. Available from: https://www.cdc.gov/vaccines/ acip/acip-scdm-faqs.html. Accessed May 5, 2020.

20. Dempsey AF, Brewer SE, Pyrzanowski J, Sevick C, O'Leary ST. Acceptability of human papillomavirus vaccines among women older than 26 years. Vaccine 2015;33:1556-61.

21. Weiss TW, Rosenthal SL, Zimet GD. Attitudes toward HPV Vaccination among Women Aged 27 to 45. ISRN Obstet Gynecol 2011;2011:670318.

22. Nichol KL, Mac Donald R, Hauge M. Factors associated with influenza and pneumococcal vaccination behavior among high-risk adults. J Gen Intern Med 1996;11:673-7.

23. Nichol KL, Lofgren RP, Gapinski J. Influenza vaccination. Knowledge, attitudes, and behavior among high-risk outpatients. Arch Intern Med 1992;152:106-10.

24. Armstrong K, Berlin M, Schwartz JS, Propert K, Ubel PA. Barriers to influenza immunization in a low-income urban population. Am J Prevent Med 2001;20:21-5.

25. Winston CA, Wortley PM, Lees KA. Factors associated with vaccination of Medicare beneficiaries in five U.S. communities: results from the racial and ethnic adult disparities in immunization initiative survey, 2003. J Am Geriatr Soc 2006;54:303-10.

26. Rosenthal SL, Weiss TW, Zimet GD, Ma L, Good $\mathrm{MB}$, Vichnin MD. Predictors of HPV vaccine uptake among women aged 19-26: importance of a physician's recommendation. Vaccine 2011;29:890-5.

27. Crane LA, Daley MF, Barrow J, et al. Sentinel physician networks as a technique for rapid immunization policy surveys. Eval Health Prof 2008;31:43-64.

28. Brtnikova M, Crane LA, Allison MA, Hurley LP, Beaty BL, Kempe A. A method for achieving high response rates in national surveys of U.S. primary care physicians. PLoS One 2018;13:e0202755. 
29. Dillman DA, Christian LM. Internet, phone, mail, and mixed-mode surveys: the tailored design method. 4th ed. Hoboken, NJ: John Wiley \& Sons, Inc.; 2014.

30. Prophylactic efficacy of a quadrivalent human papillomavirus (HPV) vaccine in women with virological evidence of HPV infection. J Infect Dis 2007;196: 1438-46.

31. Garland SM, Hernandez-Avila M, Wheeler CM, et al. Quadrivalent vaccine against human papillomavirus to prevent anogenital diseases. N Engl J Med 2007;356:1928-43.

32. Mazza D, Petrovic K, Chakraborty S. HPV vaccination of adult women: an audit of Australian general practitioners. Aust N Z J Obstet Gynaecol 2012;52:528-33.

33. Wimberly YH, Hogben M, Moore-Ruffin J, Moore SE, Fry-Johnson Y. Sexual history-taking among primary care physicians. J Natl Med Assoc 2006;98:1924-9.

34. Abbo ED, Zhang Q, Zelder M, Huang ES. The increasing number of clinical items addressed during the time of adult primary care visits. J Gen Intern Med 2008;23:2058-65.
35. Ostbye T, Yarnall KS, Krause KM, Pollak KI, Gradison M, Michener JL. Is there time for management of patients with chronic diseases in primary care? Ann Fam Med 2005;3:209-14.

36. Centers for Disease Control and Prevention. Evidence to recommendations for HPV vaccination of adults, ages 27 through 45 years. 2019. Available from: https://www.cdc.gov/vaccines/acip/recs/grade/ HPV-adults-etr.html. Accessed April 19, 2020.

37. Markowitz LE, Gee J, Chesson H, Stokley S. Ten years of human papillomavirus vaccination in the United States. Acad Pediatr 2018;18:S3-S10.

38. Chesson H. Overview of Health Economic Models of HPV Vaccination of Mid-Adults. Advisory Committee on Immunization Practices. Published 2019. Available from: https://stacks.cdc.gov/view/ cdc/82631/cdc_82631_DS1.pdf. Accessed December $5,2020$.

39. Laprise JF, Chesson HW, Markowitz LE, et al. Effectiveness and cost-effectiveness of human papillomavirus vaccination through age 45 years in the United States. Ann Intern Med 2020;172:22-9.

40. Beach MC, Sugarman J. Realizing shared decisionmaking in practice. JAMA 2019;322:811-2. 